Objectives To evaluate the mortality experience among all workers $(n=3199)$ employed at a phosphate fertiliser plant in central Florida beginning 1953 and followed through 2005.

Method All-cause, all-cancers, and cause-specific standardised mortality ratios (SMRs) were calculated with the U. S. population as referent. Lung cancer and leukaemia risks were further evaluated using conditional logistic regression. Employment duration was used as an exposure surrogate for dose-response analyses.

Results The mortality due to all causes combined ( $\mathrm{SMR}=1.07$, 95\% confidence interval $(\mathrm{CI})=1.01-1.13$, observed deaths $\mathrm{n}=$ $1124)$, lung cancer $(\mathrm{SMR}=1.25,95 \% \mathrm{CI}=1.04-1.49, \mathrm{n}=122)$, leukaemia $(\mathrm{SMR}=1.76,95 \% \mathrm{CI}=1.02-2.81, \mathrm{n}=17)$, and chronic obstructive pulmonary disease $(\mathrm{SMR}=1.45, \quad 95 \%$ $\mathrm{CI}=1.09-1.89, \mathrm{n}=54)$ were significantly elevated. All-cancer mortality was elevated $(\mathrm{SMR}=1.09,95 \% \mathrm{CI}=0.97-1.22, \mathrm{n}=$ 303) but not statistically significant for the cohort. Doseresponse modelling with adjustments for gender and race did not show statistically significant associations between employment duration (in years) and lung cancer (Odds Ratio (OR) $=0.99,95 \% \mathrm{CI}=0.97-1.02)$ or leukaemia $(\mathrm{OR}=1.01,95 \%$ $\mathrm{CI}=0.96-1.06)$ mortality.

Conclusions Findings are suggestive of increased lung cancer and leukaemia mortality from exposures encountered in the phosphate fertiliser industry. Increased employment duration, however, did not have significant associations with increased lung cancer or leukaemia mortality.

\section{THE IMPACT OF OCCUPATIONAL STRESS ON SUICIDE IDEATION OF SUBWAY DRIVERS}

Jongin Lee, Junsu Byun, Se Eun Kim, Hye-Eun Lee, Hyoung-Ryoul Kim. Department of Occupational and Environmental Medicine, Seoul St. Mary's Hospital, The Catholic University of Korea, Seoul, Republic of Korea

\subsection{6/oemed-2014-102362.230}

Objectives To investigate and manage subway drivers' mental health, we conducted a temporary mental health checkup on the subway drivers of one transportation company, and analysed the relationship between the suicide ideation and occupational stress factors.

Method The subway drivers $(\mathrm{n}=995)$ were asked to fill out a questionnaire and individual interviews were conducted afterwards. Interviews were performed using Korean Composite International Diagnostic Interview (K-CIDI). Participants were classified into two groups; suicide ideation group (SIG) and non suicide ideation group (NSIG). Socio-demographic factors, health information, occupational characteristics, Korean Occupational Stress Scale (KOSS), Davidson Trauma Scale (DTS) and Centre for Epidemiological Studies-Depression Scale (CES-D) were included in the questionnaire. Relationship between occupational stress and the suicide ideation within a year were analysed using multiple logistic regression.

Results Logistic regression model after adjusting the age showed that domains of Job demand, Insufficient job control, Interpersonal conflict, Lack of reward, Occupational climate had significant relationship with suicidal ideation. Another model that adjusted factors that was significant in descriptive statistics revealed that domains of Insufficient job control $(\mathrm{OR}=2.223)$, Interpersonal conflict $(\mathrm{OR}=2.478)$, Lack of reward $(\mathrm{OR}=2.701)$ had significant relationship with suicidal ideation.

Conclusions Three occupational stress domains of KOSS had statistically significant relationship with the suicidal ideation within a year after adjusting occupational factors that was related to it. To prevent subway drivers' suicide ideation, stress management program should be applied to this group.

\section{STUDY ON DISEASES OF THE SKIN IN AN INDUSTRIAL POPULATION OF A PETROCHEMICAL SITE IN SICILY}

${ }^{1}$ Mario Bambara, ${ }^{2}$ Massimiliano Magnano, ${ }^{3}$ Giampiero Castelli, ' Giuseppe Sole. ${ }^{1}$ Raffinerie ISAB -Lukoil, Priolo Gargallo, Siracusa-Sicily, Italy; ${ }^{2}$ stituto Archimede, Siracuse, Sicily, Italy; ${ }^{3}$ ASP Siracuse-Dermatology Department, Siracuse, Sicily, Italy

\subsection{6/oemed-2014-102362.231}

Objectives The study 'Skin Project' took place over three years (2010-2012) in a petrochemical site in Sicily, in order to identify skin diseases and the risk of pre-cancerous changes. The idea for the study came from an evaluation of mortality data for skin diseases published by the province of Siracusa in the journal 'ATLAS RERURUM cognoscere causas' 2003-2005.

Method The health service of the petrochemical company had addressed first the interest towards its employees, directing them to the screening of skin disorders and in particular towards precancerous and non precancerous forms. The first pilot to assess the eventual success of the project was launched in 2010; the public was invited to undergo a skin examination and videodermatoscopy and epiluminescence mapping, simply by calling a dedicated health service number. The first cases also showed the need to reach an agreement with the city hospital for the surgical removal of malignant growths.

The visit was carried out by a medical doctor specialising in dermatology, in this case the primary dermatologist of ASP Syracuse and heightened by the use of dermoscopy and epiluminescence. The use of non-invasive technology allowed the study to show the morphological structural of the pigmented lesions.

Results From October 2011 to October 2013, 788 dermatological and videodermatoscopy with epiluminescence have been carried out, identifying and permitting the surgical removal of 10 melanomas surface, 6 basal cell epithelioma and the identification of more than 40 individuals with dysplastic moles.

Conclusions During the medical examination carried out by the qualified doctor or dermatologist, the patient is asked to undergo a primary prevention and skin self-examination. The study showed a significant effect on the onset of skin diseases compared to the national population and correlated with sun exposure from a young age or from the high temperatures recorded in recent years in Sicily between 2006 and 2012. We report the cases of 3 person a 48 year old male that in two sessions a year apart has seen a trasformation of his nevus after the removal of melanoma surface. of a subject of 40 years old whom removed a melanoma in the region above the right eyebrow and a third case of a man 52 years old removed a melanoma of the left ear.

\section{CANCER MORTALITY AMONG STYRENE AND FIBREGLASS EXPOSED WORKERS IN THE REINFORCED PLASTIC BOATBUILDING INDUSTRY}

Avima Ruder, Alysha Meyers, Steven Bertke. Centers for Disease Control and Prevention, National Institute for Occupational Safety and Health, Cincinnati, Ohio, USA

\subsection{6/oemed-2014-102362.232}

Objectives Mortality was updaated through 2008 for 5203 workers exposed to styrene, fibreglass, and wood dust between 